\title{
Multiple Andreev reflection and giant excess noise in diffusive superconductor/normal-metal/superconductor junctions
}

\author{
T. Hoss, C. Strunk, ${ }^{*}$ T. Nussbaumer, R. Huber, ${ }^{\dagger}$ U. Staufer, ${ }^{\ddagger}$ and C. Schönenberger \\ Institut für Physik, Universität Basel, Klingelbergstraße 82, CH-4056 Basel, Switzerland
}

(Received 10 February 2000)

\begin{abstract}
We have studied superconductor/normal-metal/superconductor $(S N S)$ junctions consisting of short Au or $\mathrm{Cu}$ wires between $\mathrm{Nb}$ or $\mathrm{Al}$ banks. The $\mathrm{Nb}$ based junctions display inherent electron heating effects induced by the high thermal resistance of the NS boundaries. The Al based junctions show in addition subharmonic gap structures in the differential conductance $d I / d V$ and a pronounced peak in the excess noise at very low voltages $V$. We suggest that the noise peak is caused by fluctuations of the supercurrent at the onset of Josephson coupling between the superconducting banks. At intermediate temperatures where the supercurrent is suppressed a noise contribution $\propto 1 / V$ remains, which suggests the presence of a long-range proximity effect in the noise.
\end{abstract}

\section{INTRODUCTION}

Superconductor/normal-metal $(S N)$ interfaces of high transparency exhibit remarkably different properties for electric charge and energy transfer, respectively. Quasiparticles with energy $\epsilon$ below the energy gap $\Delta$ of the superconductor cannot enter the superconductor. This implies a high thermal resistance of the $S N$ boundary since energy is exclusively carried by the quasiparticles. ${ }^{1}$ In contrast, charge can be transmitted at $\epsilon<\Delta$ via the Andreev reflection process: An electron coming from the normal side is reflected as a hole and a Cooper pair is transferred to the superconductor. ${ }^{2}$ This should have important consequences for the energy distribution of quasiparticles in a short normal bridge connected to two superconducting reservoirs. Here we assume that the length $L$ of the bridge is larger than the thermal diffusion length $L_{T}=\sqrt{\hbar D / 2 \pi k_{B} T}$ which governs the penetration of Cooper pairs into the normal wire. For $L>L_{T}$ the supercurrent through the structure is exponentially weak.

In the case of normal reservoirs the distribution function $f(\epsilon)$ in the wire either assumes a two step shape if the inelastic scattering length $L_{i n}(\epsilon) \gg L$, or smears out into a Fermi-Dirac function with a spatially varying electron temperature if $L_{i n}(\epsilon) \ll L .^{3-5}$ The broadening of $f(\epsilon)$ has been detected by local tunneling spectroscopy, ${ }^{4}$ or by measuring the power spectral density $S_{V}(V)$ of the current noise in the junction. $^{5-7}$

In the case of superconducting reservoirs the broadening of $f(\epsilon)$ is expected to be much more dramatic when compared to normal reservoirs. In particular for small applied voltages $e V \ll \Delta$ quasiparticles have to climb up to the energy gap $\Delta$ via multiple Andreev reflections (MAR) at the two $S N$ boundaries in order to remove the deposited energy into the reservoirs. For samples shorter than the phase coherence length $L_{\phi}$ subharmonic gap structures have been observed in diffusive samples ${ }^{8}$ in the differential conductance $d I / d V$ at voltages close to $V=2 \Delta / e n$ where $n$ counts the number of reflections in the MAR cycle. Such structures have been found earlier in superconducting microbridges, tunnel junctions and ballistic $S / N / S$ point contacts. ${ }^{9}$ It is interesting to look for such long-range proximity effects also in the noise. There are theoretical ${ }^{10-13}$ and experimental ${ }^{14}$ indications that the MAR cycle transfers multiple charge quanta of magnitude $2 \Delta / V$, which should lead to an enhanced current noise at low bias voltages. For diffusive systems so far only indications of charge doubling have been reported, which points towards single Andreev reflection events. ${ }^{15}$

In this work, we address the above questions by measuring $d I / d V$ and $S_{V}$ of high transparency $\mathrm{Nb} / \mathrm{Au} / \mathrm{Nb}, \mathrm{Al} / \mathrm{Au} /$ $\mathrm{Al}$, and $\mathrm{Al} / \mathrm{Cu} / \mathrm{Al}$ junctions prepared by means of an anorganic shadow mask. ${ }^{16}$ Compared to previous studies ${ }^{17}$ we focus on junctions having a very small critical current. This allows to study the low voltage regime which is otherwise difficult to access because of heating effects.

\section{SAMPLE PREPARATION}

The samples are prepared by angle evaporation through a suspended $\mathrm{Si}_{3} \mathrm{~N}_{4}$ mask on a $\mathrm{Si}$ substrate with a $\mathrm{SiO}_{2}$ spacer layer. ${ }^{16}$ The anorganic mask avoids the previously observed deterioration of the superconducting properties of the $\mathrm{Nb}$ by outgassing of the conventional organic resist (e.g., PMMA) during evaporation of the high melting point $\mathrm{Nb}^{18}$ The $\mathrm{Si}_{3} \mathrm{~N}_{4}$ top layer is patterned by conventional electron beam lithography. Wet etching of the $800 \mathrm{~nm} \mathrm{SiO}{ }_{2}$ layer results in the desired undercut profile. The high mechanical strength of the $\mathrm{Si}_{3} \mathrm{~N}_{4}$ allows large undercuts and freely suspended brigdes of several micron length. The transition temperature of narrow $\mathrm{Nb}$ wires displayed only a minor reduction $(0.2 \mathrm{~K})$ of the superconducting transition temperature $T_{c}$ when compared to codeposited Nb films. ${ }^{16}$ Our $S N S$ devices consist of thin $(\simeq 15 \mathrm{~nm})$ normal wires $(\mathrm{Au}$ or $\mathrm{Cu})$ of $0.4-2 \mu \mathrm{m}$ length and 100-200 nm width between thick (50-200 nm) reservoirs made of $\mathrm{Nb}$ or $\mathrm{Al}$. A scanning electron micrograph of a $\mathrm{Nb} / \mathrm{Au} / \mathrm{Nb}$ sample is shown in Fig. 1.

A single $S N S$ junction and a series of 9-16 junctions together with $20 \mu \mathrm{m}$ long $N$ and $S$ wires were prepared simultaneously on the same chip. From weak localization measurements of the long wires we determined $L_{\phi}$ of the $N$ metal. The spectral density $S_{V}$ of the voltage fluctuations 


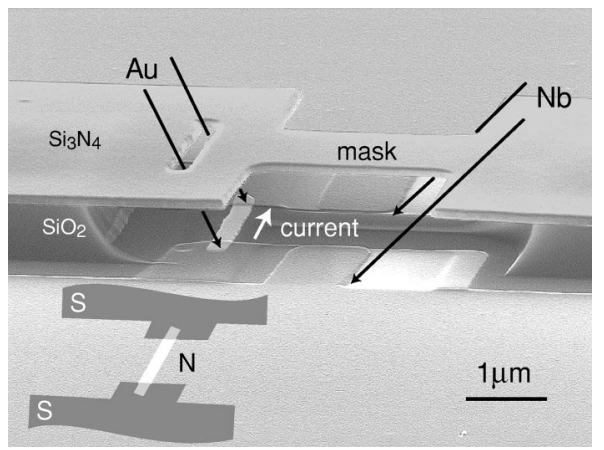

FIG. 1. Scanning electron micrograph of a typical sample viewed under a large tilt angle. The normal wire between the two $\mathrm{Nb}$ reservoirs (top and bottom) is defined through the slit in the freely suspended nitride mask. Inset: schematic of the sample layout.

across the sample is measured as a function of current bias in the frequency range between 100 and $400 \mathrm{kHz}$ with a crosscorrelation technique. ${ }^{7}$ With this technique we obtain a voltage sensitivity of $50 \mathrm{pV}^{2} / \sqrt{\mathrm{Hz}}$ with commercial roomtemperature preamplifiers. The measurements are performed in a ${ }^{3} \mathrm{He}$ cryostat which is shielded from $\mathrm{rf}$ interference by $\pi$ filters at room temperature and by a thermocoax filtering stage at the $0.3 \mathrm{~K}$ stage. The sample is put into an rf tight copper chamber at the sample temperature.

\section{NB/AU/NB JUNCTIONS}

In Fig. 2 we show the differential conductance $d I / d V$ vs applied voltage of a single Au wire of length $L=1 \mu \mathrm{m}$ between $\mathrm{Nb}$ reservoirs. The inset displays $R(T)$ for the same sample. The data are recorded using ac currents of typically 10 and $20 \mathrm{nA}$. When lowering the temperature the resistance

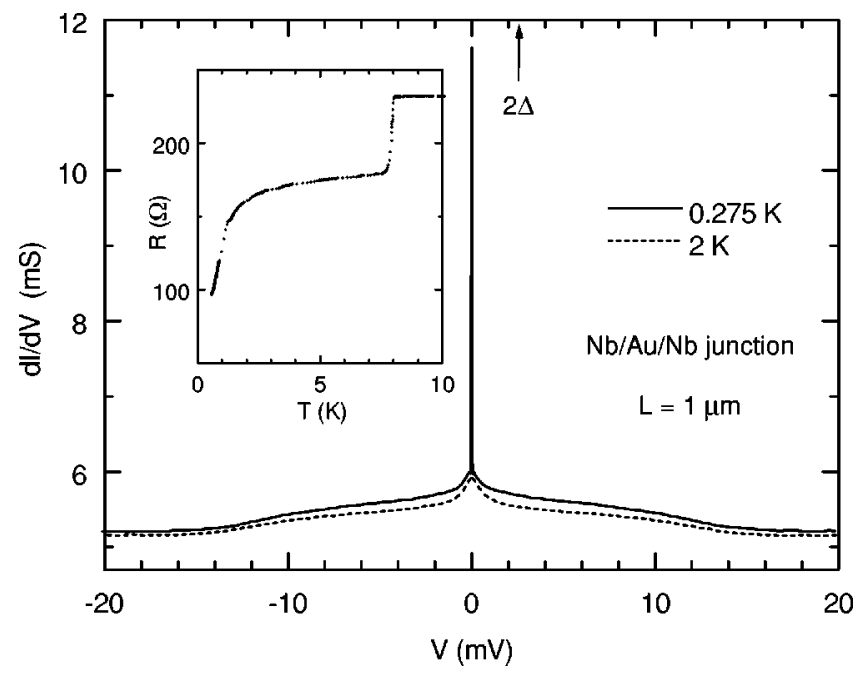

FIG. 2. Differential conductance $d I / d V$ of a single $\mathrm{Nb} / \mathrm{Au} / \mathrm{Nb}$ junction as a function of voltage $V$ for two temperatures. The sharp peak at $V=0$ indicates the rapid destruction of electron-hole coherence by a finite bias voltage. The arrow indicates $V=2 \Delta / e$ $=2.6 \mathrm{mV}$. Note the absence of subharmonic gap structures. The Au wire is $1 \mu \mathrm{m}$ long, $130 \mathrm{~nm}$ wide, and $15 \mathrm{~nm}$ thick. The thickness of the $\mathrm{Nb}$ reservoirs is $50 \mathrm{~nm}$. Inset: Resistance vs temperature for the same sample.

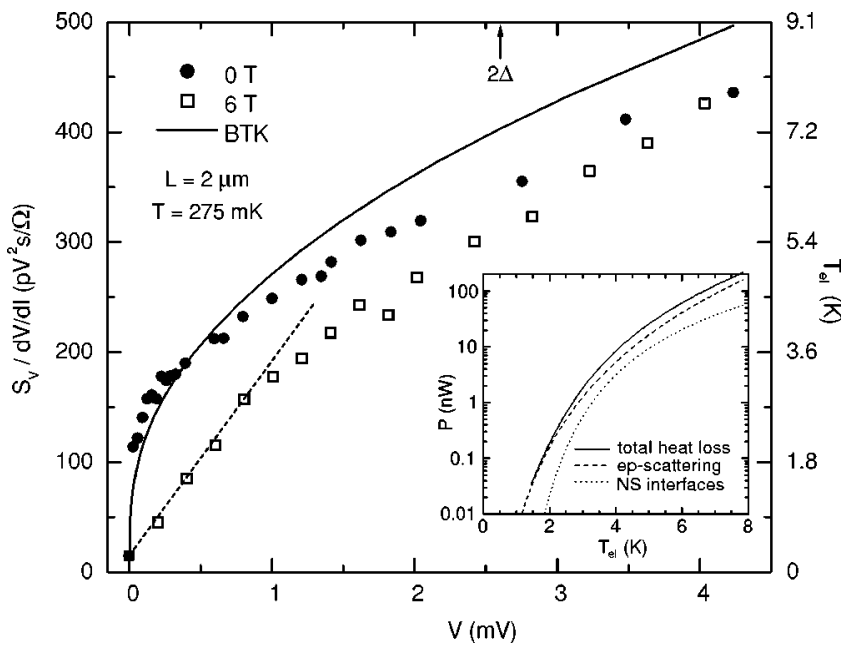

FIG. 3. Scaled excess noise $S_{V} / d V / d I$ as a function of voltage for a series of nine $\mathrm{Nb} / \mathrm{Au} / \mathrm{Nb}$ junctions of $2 \mu \mathrm{m}$ length, $200 \mathrm{~nm}$ width, and $15 \mathrm{~nm}$ thickness for normal $(\square)$ and superconducting (O) $\mathrm{Nb}$ reservoirs. The arrow indicates $V=2 \Delta / e=2.6 \mathrm{mV}$. The zero-bias resistance per junction is $39(72) \Omega$ at $B=0(6) \mathrm{T}$ and the diffusion constant of the wire is $D \simeq 68 \mathrm{~cm}^{2} / \mathrm{s}$. At $6 \mathrm{~T}$ the $\mathrm{Nb}$ reservoirs contribute significantly to the junction resistance. The right-hand scale indicates the effective electron temperature. The dashed line indicates the shot noise of noninteracting electrons in case of normal reservoirs. The solid line gives an estimate of the electron heating effect according to Eqs. (1) and (2). Inset: Electron-phonon (dashed line) and $N / S$ interface (dotted line) contributions to the cooling power as a function of electron temperature $T_{e l}$ in the wire. $T_{e l}$ in the reservoirs is assumed to remain at $0.27 \mathrm{~K}$.

first drops around $8.2 \mathrm{~K}$ which indicates the superconducting transition of the reservoirs. Further reduction of the temperature leads to a continuous decrease of $R$ which becomes more drastic below $2 \mathrm{~K}$. This proximity induced reduction of $R$ is accompagnied by a sharp peak in the differential conductance $d I / d V$ at zero bias voltage. The peak has a width of $50 \mu \mathrm{V}$ (which is close to $k_{B} T$ at $0.3 \mathrm{~K}$ ) and can be seen as the precursor of a supercurrent which emerges when $L_{T}$ becomes comparable to the wire length $L$. The peak has a height of only $10 \%$ of the normal-state resistance $R_{N}$ of the wire for $L=2 \mu \mathrm{m}$, while we find supercurrents up to $50 \mu \mathrm{A}$ for $L=0.4 \mu \mathrm{m}$ in samples with Nb banks.

In Fig. 3 we present the excess noise $S_{V}$ of a series of nine $\mathrm{Nb} / \mathrm{Au} / \mathrm{Nb}$ junctions with $2 \mu \mathrm{m}$ long Au wires. As a reference measurement, we first collected data in a perpendicular magnetic field of $6 \mathrm{~T}$ in which the $\mathrm{Nb}$ reservoirs are normal (open squares). For a direct comparison of the (effective) electron temperatures $T_{e l}$ we have normalized $S_{V}$ with $d V / d I$ (see right-hand scale). For lower voltages $V<1 \mathrm{mV}$ the measured noise falls on the $1 / 3$ reduced shot noise (dashed line). At higher voltages, additional cooling via electron-phonon scattering results in a negative curvature of $S_{V} \cdot{ }^{19}$

In the case of superconducting reservoirs (solid circles) we find a dramatic increase of $S_{V}$ in particular for the smallest voltages. The normalized excess noise rises with nearly vertical slope at $V=0$ and merges at $V \sim 2 \Delta / e$ into the $6 \mathrm{~T}$ curve. The latter is expected because for energies $\epsilon \gtrsim \Delta$ the probability of Andreev reflection rapidly vanishes. Note that $T_{e l}$ is already $\simeq 6 \mathrm{~K}$ for $V \simeq 2 \Delta / e$. From weak localization 
measurements on the long $\mathrm{Au}$ wire we infer $L_{\phi}=0.63 \mu \mathrm{m}$ at $1.3 \mathrm{~K}(0.4 \mu \mathrm{m}$ at $4.2 \mathrm{~K})$. Since $L_{\phi}$ is considerably shorter than the wire length of $2 \mu \mathrm{m}$ the MAR cycle is incoherent. This is confirmed by the absence of subharmonic gap features in $d I / d V$ (see Fig. 2).

The electron temperature in the Au wire is controlled by the power dissipation in the wire, the energy loss via quasiparticle transmission through the $S / N$ interfaces, and the electron phonon scattering in the Au wire. ${ }^{7}$ At low $T_{e l}$ the electronic heat diffusion within the Au wire is much faster than the energy loss across the interfaces so that we may assume local thermal equilibrium with a nearly constant temperature profile along the wire. The heat transfer through the interfaces can be reasonably well described in terms of a simple expression for the heat current $P_{N S}\left(T_{e l}\right)$ through the $N / S$ boundaries:

$$
P_{N S}\left(T_{e l}\right)=\frac{2}{R_{m} e^{2}} \int_{-\infty}^{\infty} \epsilon\left(f_{N}-f_{S}\right)(1-A-B) d \epsilon .
$$

This formula is analogous to the Blonder-Tinkham-Klapwijk (BTK) expression for the charge current through a point contact, where, $R_{m}$ is the normal-state resistance of the $N / S$ boundary, $f_{N}\left(T_{e l}\right)$ and $f_{S}\left(T_{B a t h}\right)$ are the Fermi functions in the wire and the reservoirs, while $A(\epsilon, Z)$ and $B(\epsilon, Z)$ are the coefficients of Andreev and normal reflection, and $Z$ is the interface parameter. We estimate $R_{m} \simeq 5 \Omega$. Within our simplified model, the cooling power via electron phonon scattering is given by

$$
P_{e p}\left(T_{e l}\right)=\left(\frac{k_{B}}{e}\right)^{2} \frac{L^{2} \Gamma}{R_{N}}\left(T_{e l}^{5}-T_{B a t h}^{5}\right),
$$

where $L$ is the length of the normal wire and $\Gamma \simeq 5$ $\times 10^{8} \mathrm{~K}^{-3} \mathrm{~m}^{-2}$ for Au. ${ }^{7}$ The parameter $\Gamma$ is related to the electron-phonon scattering rate: $\tau_{e p}^{-1}=\zeta(3) / 2 \zeta(5) D \Gamma T_{e l}^{3}$, where $\zeta(n)$ is the Riemann zeta function. ${ }^{20}$ The calculated cooling power according to Eqs. (1) and (2) is plotted as a function of $T_{e l}$ in the wire (dotted and dashed line) in the inset of Fig. 3. For simplicity we assume $Z=0$. The solid line is the sum of both contributions and corresponds to the solid line in the main Fig. 3. Finite values of $Z$ lead to a shift of the solid lines to lower cooling power and to higher electon temperatures, respectively. At intermediate temperatures both contributions are of comparable magnitude, while the electron-phonon term wins at low temperature because of the exponential cutoff of the $N / S$ interface term and at high temperature because of the strong $T_{e l}^{5}$ increase of the electron phonon term. In our geometry where the area of the $N / S$ interface is tiny $\left(200 \times 200 \mathrm{~nm}^{2}\right)$, the $N / S$ interface term is much smaller than in the related experiment on $\mathrm{Nb} / \mathrm{Al} / \mathrm{Nb}$ junctions by Jehl et al., ${ }^{15}$ who used subtractive structuring of a $\mathrm{Nb} / \mathrm{Al}$ bilayer. This may be the reason, why heating effects appear to be negligible in the latter experiment.

\section{AL/CU/AL JUNCTIONS}

It is now very interesting to look at samples, in which $L_{\phi}(\epsilon)$ remains larger than the wire length. To avoid inelastic scattering, it is necessary to keep $T_{e l}$ below $\simeq 1 \mathrm{~K}$ in the voltage range $V \leqslant 2 \Delta$. In a second set of experiments we

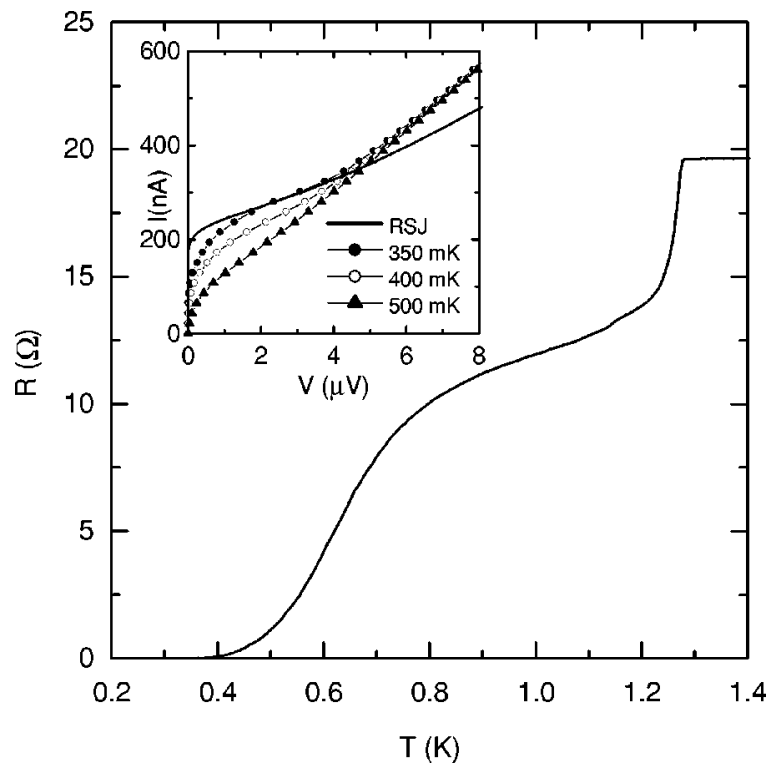

FIG. 4. Resistance vs temperature of a series of $16 \mathrm{Al} / \mathrm{Cu} / \mathrm{Al}$ junctions. The $\mathrm{Cu}$ wires are $0.9 \mu \mathrm{m}$ long, $160 \mathrm{~nm}$ wide, and $18 \mathrm{~nm}$ thick and have a diffusion constant $D \simeq 72 \mathrm{~cm}^{2} / \mathrm{s}$. The thickness of the $\mathrm{Al}$ reservoirs is $150 \mathrm{~nm}$. Inset: Current-voltage characteristics in the low-voltage region. The symbols are the experimental data and thick the solid line is a fit according to the RSJ model using $I_{c}$ $\simeq 270 \mathrm{nA}$. (Ref. 21).

replaced $\mathrm{Nb}$ by $\mathrm{Al}$, having a much smaller gap $\Delta_{A l}$. As a consequence, the energies acquired in the MAR cycle are much lower and we expect to enter the regime of coherent Andreev reflection. For the normal wire we used both $\mathrm{Au}$ and $\mathrm{Cu}$, where for $\mathrm{Cu}$ we measured a longer phase coherence length of $L_{\phi}(1.3 \mathrm{~K})=1.2 \mu \mathrm{m}$ than for $\mathrm{Au}$, where $L_{\phi}(1.3 \mathrm{~K})=0.63 \mu \mathrm{m}$. Figure 4 shows the resistance vs temperature of a series of $16 \times 1 \mu \mathrm{m}$ long $\mathrm{Al} / \mathrm{Cu} / \mathrm{Al}$ junctions. When lowering $T$ the resistance sharply drops at the transition of the reservoirs $\simeq 1.25 \mathrm{~K}$ and then continuously vanishes as the proximity effect drives the $\mathrm{Cu}$ wire into a superconducting state. This sample shows zero resistance at the lowest $T$ since the Thouless energy $E_{c}=\hbar D / L^{2}$ $\simeq 5 \mu \mathrm{eV}$ and the normal-state conductance are larger compared to the $\mathrm{Nb} / \mathrm{Au} / \mathrm{Nb}$ junctions. According to the theory by Wilhelm et al. ${ }^{17}$ the critical current $I_{c}(T)$ reads in the limit $k_{B} T \gg E_{c}$ :

$$
I_{c}(T)=\frac{3.0 \mathrm{mV} / \mathrm{K}}{R_{N} \sqrt{T_{0}}} T^{3 / 2} \exp \left(-\sqrt{T / T_{0}}\right),
$$

where $T_{0}=E_{c} / 2 \pi k_{B}$

The inset of Fig. 4 displays the measured current-voltage $(I V)$ characteristics of the same sample. The turning point of the $I V$ curves indicates $I_{c}(350 \mathrm{mK}) \approx 300 \mathrm{nA}$. With our sample parameters we estimate from Eq. (3) a critical current $I_{c}(350 \mathrm{mK}) \simeq 510 \mathrm{nA}$. This estimate is reasonably close to the measured values. On the other hand, we observe a substantial broadening of the transition such that the zero voltage state is reached only for currents $\lesssim 80 \mathrm{nA}$.

At finite temperatures a certain intrinsic broadening of the $I V$ curves is expected by virtue of thermally activated phase slips which is usually described within the resistively 
shunted junction (RSJ) model. ${ }^{21}$ Our SNS junctions are self shunted with $R_{N}$ as the shunt resistance. We observe a broadening which is much stronger than expected from the RSJ model. This is illustrated by an RSJ fit using $I_{c}=270 \mathrm{nA}$ and $T=350 \mathrm{mK}$ which is represented by the thick solid line in the inset of Fig. 4. In principle, such an enhanced broadening can be caused by external electromagnetic interference. At high frequencies this source of broadening is suppressed by our rf filtering at room temperature and the sample stage. At lower frequencies we have checked that the highest spikes in the frequency spectrum correspond to current noise below $1 \mathrm{nA} / \sqrt{\mathrm{Hz}}$, which is much lower than the critical current at $350 \mathrm{mK}$. We are therefore confident that there is an intrinsic origin of the broadening of the $I V$ curves. At voltages $V \geqslant 5 \mu \mathrm{V}$ the measured currents become larger than the fit. This is caused by the excess current induced by the Andreev reflection (see the discussion below).

Being made for tunnel junctions, a failure of the RSJ model for long $S N S$ junctions is not too surprising since it takes into account only the phase degree of freedom of the pair amplitude $F(x)=\left\langle\psi_{\downarrow} \psi_{\uparrow}\right\rangle$, while it neglects spatial variations of the absolute value $|F(x)|$. In $S N S$ junctions there is a minimum of $|F(x)|$ at the center of the $N$ wire. The minimum value of $|F(x)|$ at this "weak spot" strongly depends on the ratio $L / L_{T}$ which is reflected in the temperature dependence of $I_{c}$ at temperatures $k_{B} T \ll \Delta$ described by Eq. (3). We believe that the enhanced rounding of the $I V$ curves is related to the presence of the weak spot in the $N$ wire, which greatly facilitates phase slips in long $S N S$ junctions. Correspondingly, also the shape of $R(T)$ cannot be fitted with the RSJ formulas, since the temperature dependence of $I_{c}$ is superimposed on that of the thermal activation process. In particular, $R(T)$ does not follow a simple Arrhenius law. Broadened transitions induced by phase slip processes also occur in microbridges and long filaments made from homogeneous superconductors. The latter examples differ from $S N S$ junctions in that the main temperature dependence comes from $\Delta(T)$, which is not important at temperatures $T \ll T_{c}$.

The $d I / d V$ curves of the same sample but in a larger voltage range are presented for various temperatures in Fig. 5. Besides the supercurrent at $V=0$ we find a considerable conductance enhancement for $V<2 \Delta$. In addition, conductance peaks close to $V=2 \Delta_{A l} / n e$ are present, which we attribute to coherent MAR cycles. The peaks are rather broad and the $n=3$ peak appears even to be split. The left inset shows the scaling of the peak positions with $1 / n$. The right inset displays the temperature dependence of the $2 \Delta / e$ (i.e., $n=1)$ peak. The peak voltages nicely match the BCS curve with a slightly reduced gap. Being governed by $L_{\phi}(T),{ }^{8}$ the amplitude of the MAR features shows a relatively weak temperature dependence. This is in contrast to the supercurrent which strongly varies with temperature as expected from the exponential dependence of the Josephson coupling on $L_{T}$. Nearly identical observations have been made on $\mathrm{Al} / \mathrm{Au} / \mathrm{Al}$ junctions.

In order to further check that the peaks in $d I / d V$ are indeed related to the gap energy we compared samples with different wire resistance. The critical current of the more resistive samples was substantially smaller but the peak voltages remained nearly unaffected as demonstrated by the in-

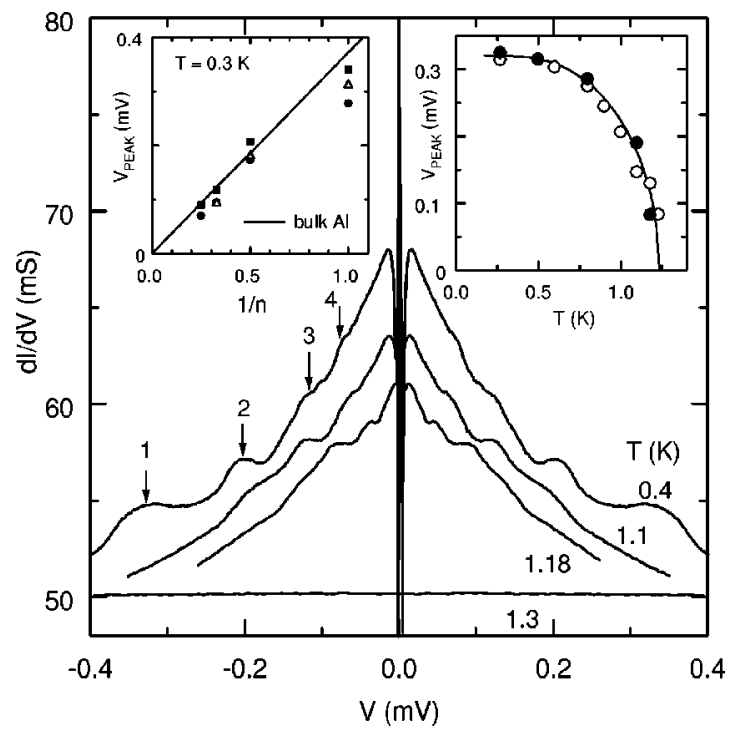

FIG. 5. Differential conductance $d I / d V$ vs voltage $V$ of the same sample as in Fig. 4 for several temperatures. The arrows indicate subharmonic gap structures corresponding approximately to integer fractions of $2 \Delta$. Left inset: Position of the conductance peaks vs $1 / n$ for three different samples. The solid line indicates the scaling for the gap of bulk Al. Right inset: Position of the $2 \Delta$ conductance peak vs temperature for two samples with different normal state conductance $(0: 50 \mathrm{mS}, \bigcirc: 29.3 \mathrm{mS})$. The solid line is a BCS fit for $2 \Delta=325 \mu \mathrm{eV}$ and $T_{c}=1.23 \mathrm{~K}$.

sets in Fig. 5. The values of the gap $\Delta(T=0)=163 \mu \mathrm{eV}$ extracted from the $2 \Delta$ peaks are slightly reduced with respect to the bulk value of $186 \mu \mathrm{eV}$. Earlier experiments on conventional $\mathrm{Nb} / \mathrm{Nb}$ point contacts ${ }^{22}$ have shown a similar suppression of the order parameter at the $n=1$ peak which was attributed to a reduction of $\Delta$ by the relatively high currents which are required to generate the voltage $2 \Delta / e$ in low Ohmic contacts with $R_{N} \simeq 20-40 \Omega$. This also contributes to deviations from the scaling of the peak voltages, i.e., $V(n=1) / V(n=2) \approx 1.6-1.8$ instead of 2 in Fig. 5. Similar effects are also visible in the data of Ref. 8.

Another important quantity is the excess current $I_{e x c}$ $=I(V)-V / R_{N}$, i.e., the enhancement of the $I V$ characteristic above the Ohmic straight line. $I_{\text {exc }}$ quantifies the integrated proximity correction to $d I / d V$ and saturates at large bias voltages $e V>2 \Delta$, where the Andreev reflection is suppressed. For superconducting point contacts with $E_{c} \gg \Delta$ the excess current is predicted to be $I_{\text {exc }}=\left(\pi^{2} / 4-1\right) \Delta / e R_{N}$ $\simeq 11 \mu \mathrm{A} \cdot{ }^{23}$ In the opposite limit of long diffusive junctions with $E_{c} \ll \Delta, I_{\text {exc }}$ is suppressed with increasing length as $1 / L$ and amounts ${ }^{24}$ to $I_{e x c}=0.82 \Delta / e R_{N} \cdot \xi^{*} / L \simeq 2.9 \mu \mathrm{A}$ where $\xi^{*}=\sqrt{\hbar D_{S} / \Delta}$ and $D_{S} \simeq 400 \mathrm{~cm}^{2} / \mathrm{s} .{ }^{25}$ When integrating the $d I / d V$ curves in Fig. 5 we find an asymptotic value of $I_{\text {exc }}$ $\simeq 3.5 \mu \mathrm{A}$, which is in acceptable agreement with the theoretical value obtained in the diffusive limit. The excess current is another feature, which is not contained in the RSJ model.

Analogous to the dc conductance, ${ }^{26}$ one may expect also in the noise different types of proximity effects occurring on the two length scales $L_{T}$ and $L_{\phi}$. In Fig. 6 we present noise data for the same sample as in Figs. 4 and 5. We indeed find a huge peak in $S_{V}$ at very low voltages around 3-4 $\mu \mathrm{V}$ 


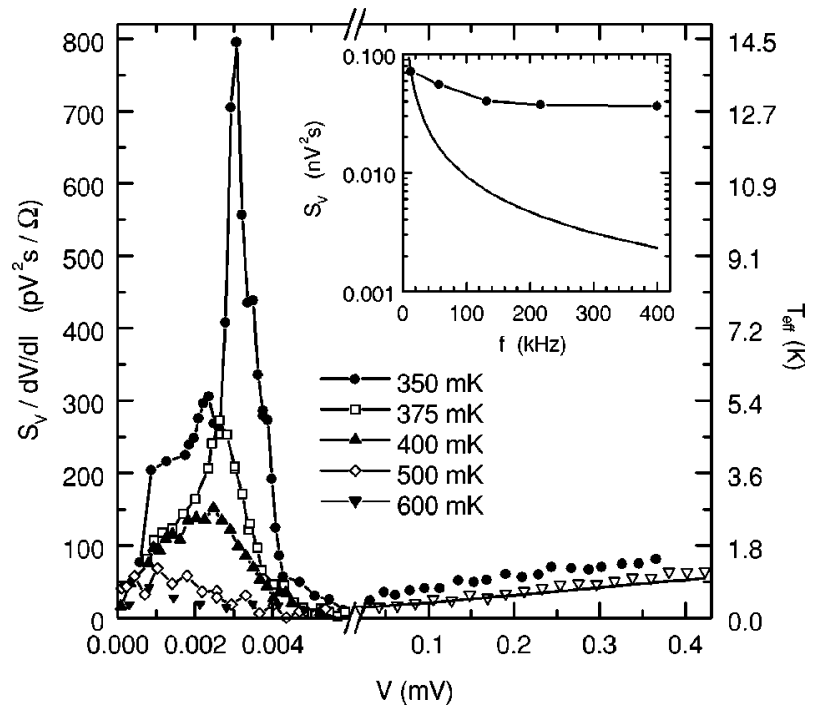

FIG. 6. Scaled excess noise $S_{V} / d V / d I$ as a function of voltage for the same device as in Fig. 5 with superconducting (O) and normal $(\nabla)$ reservoirs. The resistance per junction is $19.6 \Omega$ in the normal state. The solid line indicates the shot noise of noninteracting electrons in case of normal reservoirs. Note the expanded scale at low $V$, with data taken at $T=350,375,400,500$, and $600 \mathrm{mK}$ (from top to bottom). Inset: Amplitude of the low voltage noise peak of a similar sample as a function of frequency. The solid line corresponds to a $1 / f$ dependence.

$\approx 0.02 \Delta / e$. The noise enhancement appears in the strongly nonlinear part of $I(V)$ (inset in Fig. 4) and is frequency independent between 100 and $400 \mathrm{kHz}$ (see inset in Fig. 6). The noise peak vanishes at elevated temperatures together with the supercurrent when $L_{T}$ becomes smaller than the sample length. The nonmonotonic dependence of $S_{V}$ on $V$ is in strong contrast to the $2-\mu \mathrm{m}$-long $\mathrm{Nb} / \mathrm{Au}$ sample of Fig. 3, which shows no supercurrent at our lowest temperatures and where $S_{V}$ is monotonically rising with $V$. At higher voltages we find an enhancement of $S_{V}$ for superconducting reservoirs (full circles) with respect to normal reservoirs (open triangles). In the voltage regime $V \gtrsim \Delta$ the noise enhancement is most likely caused by heating similar to the $\mathrm{Nb} / \mathrm{Au}$ case discussed above, where the heating is even more pronounced because of the larger gap of the $\mathrm{Nb}$.

\section{DISCUSSION}

\section{A. Noise for $L_{T} \gtrsim L$}

One possible origin of the low voltage noise peak are temporal fluctuations of the critical current as previously observed in grain boundary junctions made from hightemperature superconductors. ${ }^{27}$ Such critical current fluctuations may be caused by the motion of localized defects close to the junction and should result in a $1 / f$-like frequency dependence of the voltage noise close to $I_{c}$ as well as of the normal-state resistance $R_{N}$. The latter would result in a parabolic increase of $S_{V}$ for $I>I_{c}$ which is absent in Fig. 6. At our typical measuring frequencies $f>100 \mathrm{kHz}$ the measured peak height is independent of $f$ (see the inset in Fig. 6). For $f<100 \mathrm{kHz}$ we observe a small increase of the peak amplitude which is currently not understood, but certainly incon- sistent with a $1 / f$ law. Hence $1 / f$ noise can be ruled out as the origin of the low-voltage noise peak.

Earlier experiments on shunted tunnel junctions have also revealed an increase of the noise at low voltages. ${ }^{28}$ This effect has been predicted ${ }^{29}$ to arise as a consequence of Johnson-Nyquist noise of the shunt resistor. Fluctuations at high frequencies are mixed down to low frequencies by the highly nonlinear $I V$ characteristics of the junction. Good agreement with the experiment has been found for both the noise rounding of the $I V$ curves and the excess noise. When calculating the noise according to the RSJ model using the measured $d I / d V$ and $I_{c}(T)$ in the low-voltage region for the temperatures shown in Fig. 6 we find a peak with an amplitude of $35\left(\mathrm{pV}^{2}\right) \mathrm{s} / \Omega$ at $350 \mathrm{mK}$ which is about 20 times smaller than the measured noise peak.

We believe that the noise peak is related to a strongly fluctuating supercurrent at the onset of finite voltage. As discussed already in the context of the $I V$ curves in Fig. 4, temporal fluctuations of $|F(x)|$ can be thermally excited at the weak spot in the center of the $N$ wire. These lead to large fluctuations of the supercurrent, and consequently to both large noise and unusually broad $I V$ curves. The minimum in $|F(x)|$ is the specific feature of junctions longer than $L_{T}$ and is not contained in the treatment of Refs. 10-12.

The thermally activated fluctuations of the supercurrent have to be distinguished from the fluctuations of the critical current discussed above. The latter correspond to fluctuations of the activation energy with an $1 / f$ spectrum, which are negligible at the time scale of $\mu \mathrm{s}$, where our noise measurements are usually performed.

Independent support of this interpretation is provided by the recent observation of Thomas et al. ${ }^{30}$ who found a similar thermally activated rounding of the $I V$ characteristics in InAs-based $S N S$ junctions. Their samples are also in the regime $L>L_{T}$ and the measured activation energy is typically two orders of magnitude smaller than expected from the RSJ model. In our samples, $R(T)$ is also broader than expected from the RSJ model (see the inset in Fig. 4). Thomas et al. suggest that the rounding of the $I V$ characteristics may be caused by an additional current noise, which is much larger than the Johnson noise of the device. Our work provides direct experimental evidence for such an enhanced noise at the onset of Josephson coupling in "long" $\left(E_{c} \ll \Delta\right) S N S$ contacts.

\section{B. Noise for $L_{\phi} \geqslant L$}

In contrast to the supercurrent the MAR induced subharmonic gap structure in $d I / d V$ is much less temperature dependent (see Fig. 5). This illustrates the already mentioned separation of the two length scales $L_{T}$ and $L_{\phi}$. While the noise peak in Fig. 6 vanishes for $L_{T} \leq L$ the question remains open, whether there exist also long-ranged effects in the noise. One possible effect would be the existance of multiple charge quanta $q^{*}=2 \Delta / V$ induced by of higher order MAR's. These should result in an enhanced shot noise $S_{I}$ $=2 q^{*} I$ at low voltages. ${ }^{10-12}$ A first indication for such an effect was seen in NbN based pinhole junctions. ${ }^{14}$ In order to check for MAR induced low voltage noise we have to look at higher temperatures where the supercurrent and its corresponding noise peak are suppressed. In Fig. 7 we plot the 


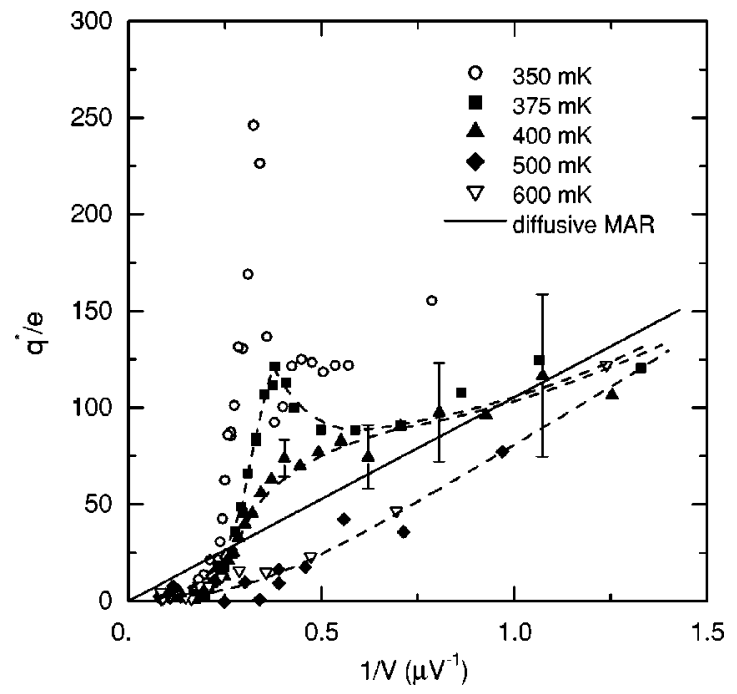

FIG. 7. Effective multiple charge $q^{*}=S_{I} / 2 e I$ as a function of $1 / V$ corresponding to the data in Fig. 6 . The solid line indicates the theoretical estimate for $q^{*}$ for the diffusive case (Ref. 12). The dashed lines are a guide to the eye. The error bars indicate the uncertainty due to the subtraction of the background noise.

effective charge $q^{*} / e=S_{I} / 2 e I$ vs $1 / V$. At low temperatures $T \lesssim 500 \mathrm{mK}$ the noise peak in $S_{V}$ is reflected also in $q^{*}$. Remarkably, $q^{*}$ rises again for even lower voltages, instead of dropping to zero as expected if the supercurrent noise is the only noise source. At higher temperatures $T \geqslant 500 \mathrm{mK}$ the peak associated to supercurrent fluctuations vanishes, but we still find a signal which rises roughly linear with $1 / V$ for $V<5-10 \mu \mathrm{V} \approx E_{c}$. At the lowest voltages all curves (dashed lines) seem to merge into a straight line with a slope only slightly lower than $\approx 0.3 \times 2 \Delta$ as predicted by the theory for the diffusive regime (solid line). ${ }^{12}$ On the other hand the theory by Naveh and Averin for the MAR noise considers very short junctions with $E_{c} \gg \Delta$. In our long $S N S$ junctions $E_{c} \ll \Delta$ is the smallest energy scale. To our knowledge, the shot noise has not yet been calculated for this case.
The measured noise corresponds to effective charge ranges up to $100 e$, which is suprisingly large since the coherence of the MAR cycle is expected to be cut off by inelastic scattering in our samples after a few Andreev reflections. From this point of view, it is already surprising that we find up to four MAR peaks in $d I / d V$. Although the magnitude and the functional dependence of the low-voltage noise in Fig. 7 are compatible with the existence of multiple charges, we cannot exclude other possibilities. Further experiments are required to clarify the origin of this longranged contribution to the noise.

\section{CONCLUSIONS}

By means of noise measurements we have shown that multiple Andreev reflections in a normal-metal wire sandwiched between two superconductors lead to substantial electron heating in the wire. The strength of this heating effect depends on the size of the gap in the superconductors. For $\mathrm{Nb}$ with a large gap the effective electron temperature raises already for small currents up to several $\mathrm{K}$, which leads to a suppression of coherent multiple Andreev reflection. For the smaller gap superconductor Al the heating is less pronounced and phase sensitive effects such as subharmonic gap structure become visible. With the onset of a proximity induced supercurrent through the normal wire a sharp noise peak appears at low voltages, which we attribute to thermally induced fluctuations of the supercurrent. When the supercurrent is suppressed at moderately elevated temperatures another contribution to the noise remains at low voltages, which indicates the existence of a long-range proximity effect in the noise.

\section{ACKNOWLEDGMENTS}

We acknowledge helpful discussions with N. Argaman, D. Averin, C. Bruder, H. Kroemer, Y. Naveh, H. Pothier, B. Spivak, E. Sukhorukov, and B. van Wees. This work was supported by the Swiss National Science Foundation.
*Present address: Institute for Experimental and Applied Physics, University of Regensburg, D-93040 Regensburg, Germany.

†Present address: Institute of Microtechnology Mainz, Department of Thin Film Technology, D-55129 Mainz, Germany.

${ }^{\ddagger}$ Permanent address: Institute for Microtechnology, University of Neuchâtel, CH-2007 Neuchâtel, Switzerland.

${ }^{1}$ A.A. Andreev, Zh. Éksp. Teor. Fiz. 46, 833 (1964) [Sov. Phys. JETP 19, 1228 (1964)].

${ }^{2}$ G.E. Blonder, M. Tinkham, and T.M. Klapwijk, Phys. Rev. B 25, 4515 (1982).

${ }^{3}$ K.E. Nagaev, Phys. Lett. A 169, 103 (1992).

${ }^{4}$ H. Pothier, S. Gueron, N.O. Birge, D. Esteve, and M. Devoret, Phys. Rev. Lett. 79, 3490 (1997).

${ }^{5}$ A. Steinbach, J.M. Martinis, and M.H. Devoret, Phys. Rev. Lett. 76, 3806 (1996).

${ }^{6}$ R.J. Schoelkopf, P.J. Burke, A.A. Kozhevnikov, D. Prober, and M.J. Rooks, Phys. Rev. Lett. 78, 3370 (1997).

${ }^{7}$ M. Henny, S. Oberholzer, C. Strunk, and C. Schönenberger, Phys. Rev. B 59, 2871 (1999).

${ }^{8}$ J. Kutchinsky, R. Taboryski, T. Clausen, C.B. Sørensen, A. Kris- tensen, P.E. Lindelof, J. Bindslev-Hansen, C. Schelde Jacobsen, and J.L. Skov, Phys. Rev. Lett. 78, 931 (1997).

${ }^{9}$ P.E. Gregers-Hansen, E. Hendricks, M.T. Levinsen, and G.R. Pickett, Phys. Rev. Lett. 31, 524 (1973); T.M. Klapwijk, G.E. Blonder, and M. Tinkham, Physica B \& C 109\&110B, 1657 (1982); W.M. van Huffelen, T.M. Klapwijk, D.R. Heslinga, M.J. deBoer, and N. van der Post, Phys. Rev. B 47, 5170 (1993); A.W. Kleinsasser, R.E. Miller, W.H. Mallison, and G.B. Arnold, Phys. Rev. Lett. 72, 1738 (1994); E. Scheer, P. Joyez, D. Esteve, C. Urbina, and M.H. Devoret, ibid. 78, 3535 (1997).

${ }^{10}$ D. Averin and H. Imam, Phys. Rev. Lett. 76, 3814 (1996).

${ }^{11}$ J.C. Cuevas, A. Martín-Rodero, and A. Levy-Yeyati, Phys. Rev. Lett. 82, 4086 (1999)

${ }^{12}$ Y. Naveh and D.V. Averin, Phys. Rev. Lett. 82, 4090 (1999).

${ }^{13}$ E.V. Bezuglyi, E.N. Bratus, V.S. Shumeiko, and G. Wendin, Phys. Rev. Lett. 83, 2050 (1999).

${ }^{14}$ P. Dieleman, H.G. Bukkems, T.M. Klapwijk, M. Schicke, and K.H. Gundlach, Phys. Rev. Lett. 79, 3486 (1997).

${ }^{15}$ X. Jehl, P. Payet-Burin, C. Baraduc, R. Calemczuk, and M. Sanquer, Phys. Rev. Lett. 83, 1660 (1999). 
${ }^{16}$ T. Hoss, C. Strunk, and C. Schönenberger, Microelectron. Eng. 46, 146 (1999).

${ }^{17}$ H. Courtois, Ph. Gandit, and B. Pannetier, Phys. Rev. B 52, 1162 (1995); F.K. Wilhelm, A.D. Zaikin, and G. Schön, J. Low Temp. Phys. 106, 305 (1997).

${ }^{18}$ Y. Harada, D. Haviland, P. Delsing, C.D. Chen, and T. Claeson, Appl. Phys. Lett. 65, 636 (1994).

${ }^{19}$ M. Henny, H. Birk, R. Huber, C. Strunk, A. Bachtold, M. Krüger, and C. Schönenberger, Appl. Phys. Lett. 71, 773 (1997).

${ }^{20}$ F.C. Wellstood, C. Urbina, and J. Clarke, Phys. Rev. B 49, 5942 (1994).

${ }^{21}$ V. Ambegaokar and B.I. Halperin, Phys. Rev. Lett. 22, 1364 (1969).

${ }^{22}$ K. Flensberg and J. Bindslev Hansen, Phys. Rev. B 40, 8693 (1989).

${ }^{23}$ A. Bardas and D.V. Averin, Phys. Rev. B 56, R8518 (1997).

${ }^{24}$ A.F. Volkov, A.V. Zaitev, and T.M. Klapwijk, Physica C 210, 21 (1993).
${ }^{25}$ Here we assume perfectly transparent interfaces. The coherence length on the superconducting side enters because of the modulation of the order parameter of the superconductor at the $N S$ interface.

${ }^{26}$ H. Courtois, Ph. Gandit, D. Mailly, and B. Pannetier, Phys. Rev. Lett. 76, 130 (1996).

${ }^{27}$ M. Kawasaki, P. Chaudhari, and A. Gupta, Phys. Rev. Lett. 68, 1065 (1992); A. Marx, U. Fath, W. Ludwig, R. Gross, and T. Amrein, Phys. Rev. B 51, 6735 (1995).

${ }^{28}$ C.M. Falco, W.H. Parker, S.E. Trullinger, and P.K. Hansma, Phys. Rev. B 10, 1865 (1974); R.H. Koch, D.J. Van Harlingen, and J. Clarke, ibid. 26, 74 (1982).

${ }^{29}$ K.K. Likharev and V.K. Semenov, Pis'ma Zh. Éksp. Teor. Fiz. 14, 625 (1972) [JETP Lett. 15, 442 (1972)]; R.H. Koch, D.J. Van Harlingen, and J. Clarke, Phys. Rev. Lett. 45, 2132 (1980).

${ }^{30}$ M. Thomas, H.-R. Blank, K.C. Wong, H. Kroemer, and E. Hu, Phys. Rev. B 58, 11676 (1998). 